



Natlonal Bureau of Standards

IIbrary, N.W. Blag

SEP 121963

PB 161584

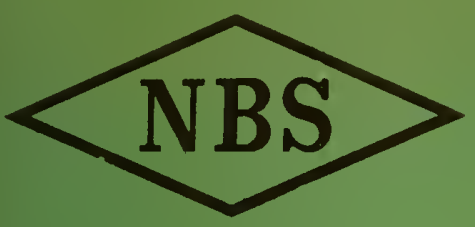

Eechnical Note

83

\section{ON THE SCATTERING OF $\gamma$ RAYS BY NUCLEI}

U. S. DEPARTMENT OF COMMERCE NATIONAL BUREAU OF STANDARDS 


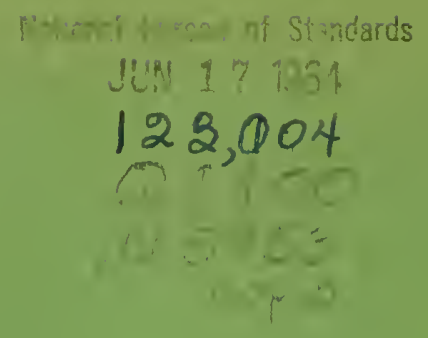

THE NATIONAL BUREAU OF STANDARDS

\section{Functions and Activities}

The functions of the National Bureau of Standards are set forth in the Act of Congress, March 3, 1901, as amended by Congress in Public Law 619, 1950. These include the development and maintenance of the national standards of measurement and the provision of means and methods for making measurements consistent with these standards; the determination of physical constants and properties of materials; the development of methods and instruments for testing materials, devices, and structures; advisory services to government agencies on scientific and technical problems; invention and development of devices to serve special needs of the Government; and the development of standard practices, codes, and specifications. The work includes basic and applied research, development, engineering, instrumentation, testing, evaluation, calibration services, and various consultation and information services. Research projects are also performed for other government agencies when the work relates to and supplements the basic program of the Bureau or when the Bureau's unique competence is required. The scope of activities is suggested by the listing of divisions and sections on the inside of the back cover.

\section{Publications}

The results of the Bureau's work take the form of either actual equipment and devices or published papers. These papers appear either in the Bureau's own series of publications or in the journals of professional and scientific societies. The Bureau itself publishes three periodicals available from the Government Printing Office: The Journal of Research, published in four separate sections, presents complete scientific and technical papers; the Technical News Bulletin presents summary and preliminary reports on work in progress; and Basic Radio Propagation Predictions provides data for determining the best frequencies to use for radio communications throughout the world. There are also five series of nonperiodical publications: Monographs, Applied Mathematics Series, Handbooks, Miscellaneous Publications, and Technical Notes.

Information on the Bureau's publications can be found in NBS Circular 460, Publications of the National Bureau of Standards (\$1.25) and its Supplement (\$1.50), available from the Superintendent of Documents, Government Printing Office, Washington 25, D.C. 


\section{NATIONAL BUREAU OF STANDARDS Eechnical Note}

83

NOVEMBER 1960

\section{ON THE SCATTERING OF \\ $\gamma$ RAYS BY NUCLEI}

U. Fano

NBS Technical Notes are designed to supplement the Bureau's regular publications program. They provide a means for making available scientific data that are of transient or limited interest. Technical Notes may be listed or referred to in the open literature. They are for sale by the Office of Technical Services, U. S. Department of Commerce, Washington 25, D. C.

DISTRIBUTED BY

UNITED STATES DEPARTMENT OF COMMERCE OFFICE OF TECHNICAL SERVICES

WASHINGTON 25, D. C.

Price 75 Cents 
Preface

This report consists, in essence, of a number of exercises and of discussions of specific topics, which were developed in connection with the experimental program of E. G. Fuller and E. V. Hayward.

TABLE OF CONTENTS

Page

1. Introduction . . . . . . . . . . . . . . 1

2. General Formulas . . . . . . . . . . . . 2

3. The Geometrical Factor . . . . . . . . . . 7

4. Polarizability Analysis in Terms of Intermediate States................. . . . 13

5. Polarizability Analysis in the Bohr-Mottelson Model . . 16

6. Core Polarizability in the Presence of a "Spectator" Particle................. . . 21

7. Appendix . . . . . . . . . . . . . . . 23 
On the Scattering of $y$ Rays by Nuclei

U. Fano

The theory of scattering by electric dipole interaction . is developed by tensorial techniques, which permit an early separation of geometric and dynamic factors. The geometric relationships are formulated in terms of variables that represent arbitrary partial polarization of the incident and scattered $y$ rays. The relevant dynamic properties of a nucleus are represented by a scalar, a vector, and a quadrupole polarizability. These polarizabilities correspond respectively to the values 0,1 , and 2 of the quantum number $j$ which indicates the angular momentum transfer in the scattering process. The analysis of scattering according to angular momentum transfer is compared to the ordinary theory of angular distributions. The nuclear polarizability is discussed from the standpoint of different models. The magnitudes of the three polarizabilities can be determined by experiments with unpolarized nuclei but with some degree of circular polarization of the $y$ rays; linear polarization contributes no additional information. Nuclear polarization is required to determine the phases of the polarizabilities.

\section{INTRODUCTION}

The theory of elastic or inelastic $\gamma$-ray scattering by nuclei has been treated by many authors. ${ }^{1}$ It is equivalent, in essence, to the theory of the scattering of light by molecules, which has been presented in a comprehensive article by Placzek. 2 The geometrical aspects of this and of related phenomena can be treated in a compact form by means of techniques of tensorial algebra that have been described in recent

$\overline{1}$

This process is a special case of the general theory of angular correlation. Particular aspects of the $(\boldsymbol{Y}, \boldsymbol{Y})$ process for deformed nuclei have been treated by A. M. Baldin, Zh. Exp. Theor. Phys. 37, 202 (1959), trans1. JETP 10, 142 (1960), Z. Maric and P. Möbius, Nuclear Phys., 10, 135 (1959), and E.G.Fuller and E. V. Hayward, Phys. Rev。 Letters, $\underline{1}$, 1507 (1958).

${ }^{2}$ G. Placzek, Marx Handb. der Radiologie, Vol. 6, part 2, p. 305 (1934). 
works, ${ }^{3}$ which wil1 be referred to as FR59 and F60, respectively。 An application of these techniques to various aspects of $\gamma$-ray nuclear scattering is presented in the following sections.

The treatment will be limited to the electric dipole interaction, which gives the main contribution to the scattering and is most prominent in the "giant resonance" range of photon energies (10-20 Mev). The treatment will include the effects of $\boldsymbol{\gamma}$-ray polarization and can be easily extended to take into account nuclear polarization or alignment.

\section{GENERAL FORMULAS}

Let us define:

$\underline{r}=$ radius vector from the center of mass of a nucleus to the center of its charge,

$A_{m} A^{\prime}=$ unit polarization vectors of the incident and scattered radiation,

$0_{-}=\left[\mathrm{H}-\mathrm{E}_{i}-\hbar \omega\right]^{-1}=$ reciprocal of resonance denominator pertaining to intermediate nuclear state following photon absorption, with $\mathrm{H}=$ Hamiltonian operator of, nucleus, $E_{i}=$ ground state energy of nucleus, $\hbar \omega=$ energy of incident radi$0_{+}=\left[H-E_{i}+\hbar \omega^{\prime}\right]^{-1}=$ reciprocal of resonance denominator applicable when emission of the "scattered" photon energy $\hbar \omega^{\prime}$ precedes the absorption of the incident photon; $\omega^{\prime}=\omega$ for elastic scattering.

3. Fano and G. Racah, Irreducible Tensorial Sets, Academic Press, (1959). U. Fano, J. Mathem. Physics, 1,417 (1960). 
The scattering operator may then be expressed, in the electric dipole approximation, as

$$
S=\underset{m}{r} \cdot A_{m}^{\prime} O_{-} \underset{m}{r} \cdot A_{m}^{A}+\underset{m}{r} \cdot A_{m} O_{+} \underset{m}{r} \cdot A_{m}^{\prime}
$$

According to the conventions of FR59, P. 22-23, the standard or contrastandard sets of vector or tensor components will be represented by letters with shill across in typing or in German type in print with an appropriate superior index. In this notation, (1) becomes

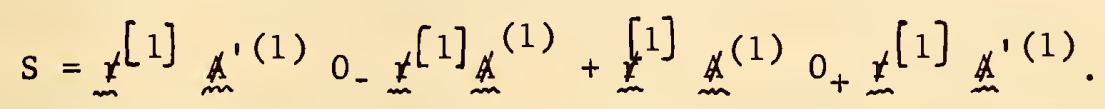

The parts of this operator that pertain to the radiation can be separated from those pertaining to the nucleus by the recoupling procedure given at the end of p. 47 of FR. This yields

$$
S=\sum_{j=0}\left[\underline{x}^{[1]} \times\left(0_{-}+(-1)^{j} 0_{+}\right) \underline{m}^{[1]}\right][j]\left[\underline{m}^{(1)} \times \underline{m}^{(1)}\right]^{(j)}
$$

The index $\mathrm{j}$ indicates the angular momentum transferred in the interaction (it was called $j_{t}$ on p. 114 of FR59). The first factor on the right side of (2) may be called the $2^{j}$-pole polarizability operator of the nucleus, and indicated by

$$
{ }_{m}^{p}[j]=\left[\underset{x}{x}[1]_{x}\left(0_{-}+(-1)^{j} 0_{+}\right) \underset{m}{[1]}\right][j] \text {. }
$$

Since $j=0,1,2$, we distinguish a scalar, a vector, and a quadrupole polarizability; the vector polarizability vanishes in the static case, since $0_{-}-0_{+}=0$ for $\omega^{\prime}=\omega=0$, and it may often be disregarded as will be seen in Sec, 4 and 5 .

Following Chap.18 of FR59 we consider here reduced sets of matrix elements of the operator $S$, between the initial and final nuclear states whose angular momentum quantum numbers $I_{i}$ and $I_{f}$ may be equal or different. Because the form (2) of $S$ coincides with (18.6) of FR59, its 
reduced sets of matrix elements are given in accordance with (18.7) by $^{4}$

$$
\left[\left(I_{f}|S| I_{i}\right)\right]_{m}^{(j)}=(2 j+1)^{-1 / 2}\left(I_{f} \| P\left[I _ { i } \left[j I_{m}\left[\alpha^{(1)} \times \underline{m}^{(1)}\right]^{(j)},\right.\right.\right.
$$

where the second factor on the right is a reduced matrix element (Eq. 14.4 of FR59) of the $2^{j}$-pole polarizability. This reduced matrix element vanishes whenever "triangular" selection rules, such as $\left|I_{i}-j\right| \leqslant I_{f} \leqslant I_{i}+j$ are violated; in particular $i$ ts vanishes for $I_{i}=0$ unless $I_{f}=j$.

If the scatterer nucleus is unpolarized, the desired scattering cross section $\sigma$ is proportional to the squared modulus of the matrix element of $\mathrm{S}$ between an initial and a final state, summed (or averaged) over the magnetic quantum numbers of these states. Application of (18.10) and (18.19) of FR59 and of (3) above yields

$$
\begin{aligned}
& \frac{\mathrm{d} \sigma}{\mathrm{d} \Omega}=\mathrm{C} \quad \Sigma_{\mathrm{m}_{i}, \mathrm{~m}_{\mathrm{f}}}\left|\left(\mathrm{I}_{\mathrm{f}} \mathrm{m}_{\mathrm{f}}|\mathrm{s}| \mathrm{I}_{i} \mathrm{~m}_{i}\right)\right|^{2}= \\
& =C \Sigma_{j}\left[\left(I_{f}|S| I_{i}\right)\right]^{(j) *}\left[\left(I_{f}|S| I_{i}\right)\right]_{m}^{(j)}= \\
& =C \sum_{j}(2 j+1)^{-1}\left|\left(\dot{I}_{f}\left\|P^{[j]}\right\| I_{i}\right)\right|^{2}
\end{aligned}
$$

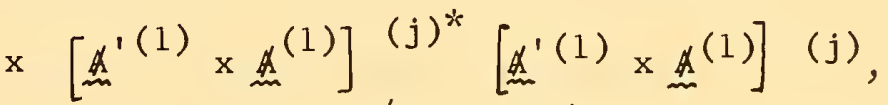

$$
\begin{aligned}
& c=\frac{e^{4}}{c^{4}} \frac{\omega \omega^{4}}{2 I+1}
\end{aligned}
$$

As a result of the expansion of the interaction in Eq. (2), all the geometrical elements of the $q$-ray angular distribution and polarization

4 The factor corresponding to $(2 j+1)^{-1 / 2}$ was omitted by mistake in (18.7) and (18.9) as printed in FR59. 
are embodied in the last factor of (4), namely, in the hermitian products of $\left[\alpha^{\prime}(1) \times{ }^{(1)}\right](j)$ by itself, for $j=0,1,2$. The nuclear properties, represented by the $2^{j}$-pole polarizabilities, enter as weight factors in the summation of the hermitian products of different degrees. If the nuclear orientation were observed before and/or after the scattering, the calculation would proceed in clese analogy to the derivation of (4). The invariant product of polarization vectors in (4) would be replaced by an invariant product of the polarization vectors and of the $2^{\mathrm{K}}$-pole tensors that describe the nuclear polarization. This new product contains a factor $\left[\left[x^{\prime(1)} \times \alpha^{(1)}\right]\left(j^{\prime}\right) * x\left[j^{\prime}(1) \times(1)\right](j)\right](K)$, with $j^{\prime} \neq j$. Accordingly the $\Sigma_{j}$ in (4) would be replaced by a $\Sigma_{j, j}$ and would include. "rectangular" polarizability factors ( $\left.I_{f} \| P[j]_{\|} I_{i}\right)^{*}$ $x\left(I_{f}\|P[j]\| I_{i}\right)$, besides the quadratic factor $\left|\left(I_{f}\|P[j]\| I_{i}\right)\right|^{2}$ which appears in (4). This result is noteworthy: Whereas the scalar, vector and tensor polarizabilities give separate contributions the cross section (4), so that their magnitudes can be determined by experiments with unpolarized nuclei as shown in the next section, the scattering by polarized nuclei depends on interference effects among the polarizabilities so that its measurement can provide the relative phases of the different polarizabilities.

The analysis of the scattering process carried out in this section differs from the analysis that underlies the familiar theories of angular correlation in nuclear physics. We have emphasized the angular momentum transferred from the radiation to the nucleus in the complete scattering process, and expressed the relevant nuclear properties in 
terms of $2^{j}$-pole polarizabilities. This analysis is represented by the angular momentum diagram in figure 1 , where $\mathrm{Lm}_{i}$ and $\mathrm{L}_{\mathrm{s}}$ represent the angular momenta of the incident and scattered radiation, respectively. (In the electric dipole approximation we have, of course, $L_{i}=L_{s}=1$. ) The triangle with sides ( $\mathrm{L}_{i}, \mathrm{~L}_{\mathrm{S}}, j$ ) in figure la corresponds to the geometrical factor in eq. (4), the triangle $\left(I_{i}, I_{f}, j\right)$ to the nuclear polarizability factor. Figure lb represents, instead, the analysis of angular momentum relationships that is performed in the usual theory of angular correlations. This analysis emphasizes the angular momentum $I$ of the intermediate nuclear state and deals with the absorption and reemission as separate stages of the scattering process; the separate stages correspond, respectively, to the triangles ( $I_{i}, L_{i}, I$ ) and $\left(I, L_{m}, I_{f}\right)$. The treatment of FR59 develops primarily the analysis corresponding to Figure $1 \mathrm{~b}$ which leads to the angular correlation formula (19.11), and deals only briefly with the analysis corresponding to Figure la which leads to equations (19.12, (19.13). The equivalence between the two types of formulas is established by a geometrical

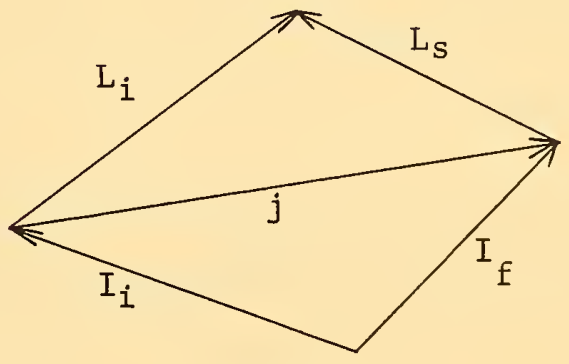

Figure 1a

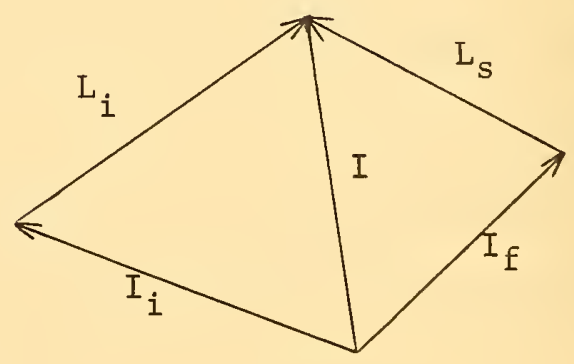

Figure 1b 
identity due to Biedenharn (FR59, P. 114 and Appendix I), and is illustrated in the Appendix of this paper. Note that the analysis according to angular momentum transfer is particularly convenient in the treatment of scattering which may result from the alternative sequences absurptionemission and emission-absorption, represented by the two terms on the right of eq. (1). (Diagrams representing the emission-absorption sequence differ from those of Figure 1 by the interchange of $\underset{\sim}{L_{i}}$ and $\mathrm{L}_{S^{*}}$ ) The interference between the contributions of the two sequences is readily represented by the expression of the $2^{j}$-pole polarizability in eq. (2).

\section{THE GEOMETRICAL FACTOR}

The product of polarization vectors in (4) is expressed in ordinary (non-standard) vector notation by

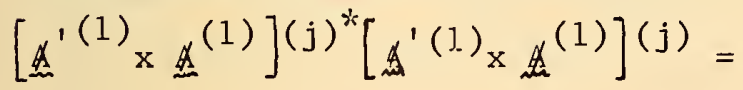

$$
\begin{aligned}
& = \begin{cases}(1 / 3)\left(\mathbb{m}^{\prime} \cdot A\right)^{2} & \text { for } j=0, \\
(1 / 2)||_{m}^{A} \times\left.\underset{m}{A}\right|^{2}=(1 / 2)\left[1-\left(A^{\prime} \cdot A\right)^{2}\right] & \text { for } j=1, \\
(1 / 2)\left[1+(1 / 3)\left(A^{\prime} \circ A\right)^{2}\right] & \text { for } j=2\end{cases}
\end{aligned}
$$

This formula is quite suitable for direct evaluation in two extreme cases, namely: (1) A and $\underset{m}{A}{ }_{m}^{\prime}$ have definite orientations, i.e., the incident $\gamma$ rays have a complete linear polarization and the scattered radiation is observed with an ideal analyzer for linear polarization (this case is unrealistic), or (2) the incident $\gamma$ rays are unpolarized and no polarization analysis is made after scattering (this occurs most frequently). In case 2) one averages over two perpendicular orientations of $\mathrm{A}$ and sums over two perpendicular orientations of $\mathrm{A}_{\mathrm{m}}^{\prime}$, thereby obtaining the following values of (5) 


$$
\begin{array}{ll}
(1 / 6)\left(1+\cos ^{2} \theta\right), & \text { for } j=0, \\
(1 / 4)\left(2+\sin ^{2} \theta\right), & \text { for } j=1, \\
(1 / 12)\left(13+\cos ^{2} \theta\right), & \text { for } j=2,
\end{array}
$$

where $\theta$ is the angle between the directions of incidence and of scattering .

A partially polarized incident beam may be represented by the incoherent superposition of two components with orthogonal polarization vectors $\mathrm{A}_{1}$ and $\mathrm{A}_{2}$ and relative intensities $\mathrm{p}_{1}$ and $\mathrm{p}_{2}=1-\mathrm{p}_{1}$. (The degree of polarization is, then, $\mathrm{p}_{1}-\mathrm{p}_{2}$.) Polarization analysis of the scattered radiation may be similarly represented by a maximum response efficiency $e_{1}$ for detection of $\gamma$ rays with a certain polarization $A_{1}^{\prime}$ and by a minimum efficiency $e_{2}$ for $\gamma$ rays with the orthogonal polarization $\mathrm{A}_{2}^{\prime}$. (The degree of selectivity of the analyzer is, then, $\left(e_{1}-e_{2}\right) /$ $\left(e_{1}+e_{2}\right)$.) In this general case one must evaluate the expression

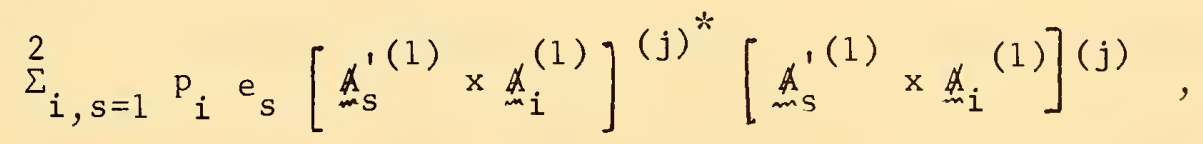

which reduces to (6) when $p_{1}=p_{2}=1 / 2$ and $e_{1}=e_{2}=1$. Notice that $\mathrm{A}_{\mathrm{m}}^{\prime} \cdot \mathrm{A}$ in (5) must be replaced by $\left|\mathrm{A}_{\mathrm{m}}^{\prime} \cdot \mathrm{A}\right|$ when the basic polarizations are circular or elliptical, in which case the vectors $\mathrm{A}_{\mathrm{i}}$ and/or $\mathrm{A}_{\mathrm{m}}^{\prime}$ are complex.

Alternatively, the distribution of scattered $q$ rays can be analyzed, with regard to both direction and polarization, by the general procedure of harmonic analysis given in Chapter 19 of FR59. This procedure represents the kind and degree of polarization of the incident or scattered $\gamma$ rays by means of variables, such as the Stokes parameters, which 
are always observable whereas the polarization vectors $\underset{m}{4}$ and $\mathrm{A}_{\mathrm{m}}^{\prime}$ are observable only for fully polarized radiation. These variables are, in essence, mean values of products of polarization vector components,

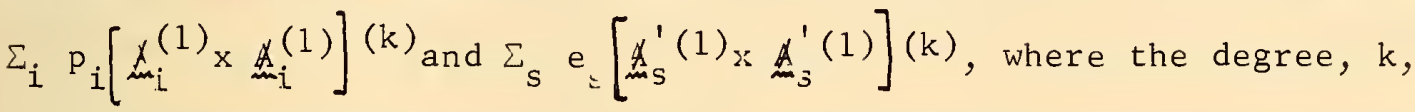
of the product ranges from 0 to 2 . The geometrical factor (5) is expressed in terms of these products by a recoupling transformation. With reference to FR59, p. 29, p. 37 and eq. (11.10) one finds that

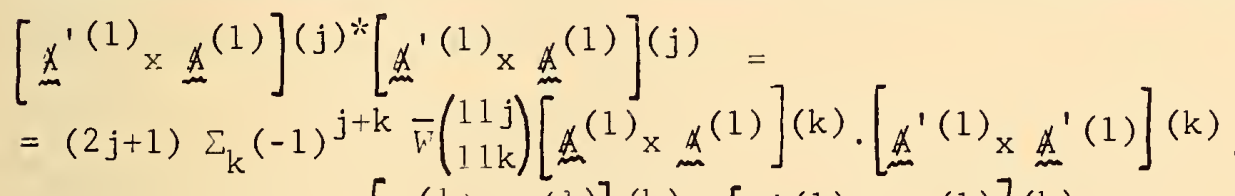
The product $\left[\AA^{(1)} \times \AA^{(1)}\right](k) \cdot\left[A^{(1)} \times{A^{\prime}}^{(1)}\right](k)$ in (7) is a harmonic function of degree $k$ of the Euler angles between frames of reference attached to the polarizer of the incident radiation and to the analyzer of the scattered radiation (FR59, Chap. 6). Accordingly the summation over $k$ in (7) represents a harmonic expansion of the angular and polarization distribution of the scattered radiation. This harmonic expansion is obtained automatically in the theories that analyze the distribution according to the coupling scheme of Figure 1b, as emphasized in Chap. i9 of FR59. When the analysis develops according to the coupling scheme of Figure 1 a, as was done in this paper, the harmonic expansion results from a recoupling transformation and takes the form of (19.13) of FR59, of which the right hand side of (7) is a special case. Note that the $\bar{W}$ coefficient in ( 7 ) is the same as one encounters when calculating the $2^{k}$-pole interaction of two p-orbitals (with $l=1$ ) coupled with a resultant quantum number $j$. 
We still have the task of obtaining an explicit expression for the scalar product on the right hand side of ( 7 ) properly averaged over the polarization intensities and detector efficiencies $p_{i}$ and $e_{S}$. (This task has not been carried out in the examples of FR59, Chap. 19, where the final results were expressed in terms of density and detector matrices and of $\not$ functions.) In this connection, it is not always convenient to characterize the polarizations by the vectors $\mathrm{A}$ and $\mathrm{A}_{\mathrm{m}}^{\prime}$ because these vectors are, in general, complex and their real and imaginary parts merely represent a pair of conjugated half-diameters of the polarization ellipse. Notice also that the polarization vector $\mathrm{A}_{\mathrm{m}}^{\prime}$ which appears in cross section formulas is not properly a characteristic of the scattered radiation but that it characterizes the analyzer detector whose probability of response is being calculated; similarly A may be regarded as a property of the polarizer that has prepared the incident beam. We shall express the cross section in terms of the following parameters :

a) the degrees of linear and circular polarization of the incident

$$
\text { rays, } P_{l}=\left|A_{m 1} \cdot A_{m}\right|\left(p_{1}-p_{2}\right) \text { and } P_{c}=\left|A_{m 1} \times \underset{m}{A}\right|\left(p_{1}-p_{2}\right) \text {; }
$$

b) the corresponding selectivities of the analyzer for linear and circular polarization of the scattered $\gamma$ rays, $Q=\left|A_{1}^{\prime} \cdot A_{1}^{\prime}\right|\left(e_{1}-e_{2}\right) /\left(e_{1}+e_{2}\right)$ and $Q_{C}=\left.\right|_{m} ^{A} A_{1}^{\prime} \times A_{m}^{\prime} * *\left(e_{1}-e_{2}\right) /\left(e_{1}+e_{2}\right)$,

c) the total response efficiency $\xi=e_{1}+e_{2}$, which constitutes only a normalization constant (simple summation over two alternative polarizations yields $\xi=2$ ), 
d) the angle $\theta$ between the directions of incidence and scattering,

e) the angle $\psi$ from the plane of incident linear polarization, identified by the polarizer orientation, to the plane of scattering,

f) the angle $\varphi$ from the plane of scattering to the plane of linear polarization selected by the analyzer.

We write, then, in terms of these variables

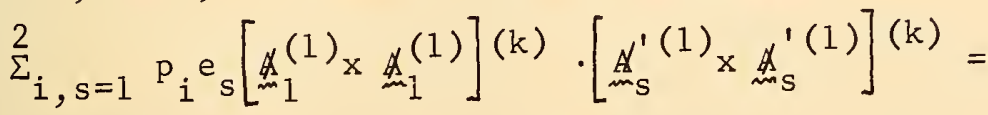

$$
\begin{aligned}
& =\xi R_{k}\left(P_{\ell}, P_{c}, Q_{\ell}, Q_{c}, \psi, \theta, \varphi\right) .
\end{aligned}
$$

The functions $R_{k}$ can be worked out in the real-standard representation of $F 60$, and are in fact obtained by setting $I_{0}=E_{0}=1$ in (26) and (27) of F60. They are

$$
\begin{aligned}
\mathrm{R}_{0} & =1 / 3 \\
\mathrm{R}_{1} & =(1 / 2) \mathrm{P}_{c} \mathrm{Q}_{c} \cos \theta \\
\mathrm{R}_{2} & =(1 / 12)\left(3 \cos ^{2} \theta-1\right)-(1 / 4)\left(\mathrm{P}_{\ell} \cos 2 \psi+Q_{\ell} \cos 2 \varphi\right) \sin ^{2} \theta+ \\
& +(1 / 4) \mathrm{P}_{\ell} \mathrm{Q}_{\ell} \cos 2 \psi \cos 2 \varphi\left(1+\cos ^{2} \theta\right)-\mathrm{P}_{\ell} \mathrm{Q}_{\ell} \sin 2 \psi \sin 2 \varphi \cos \theta .
\end{aligned}
$$

Expressing the coefficient on the right side of (7) as a matrix

$$
M_{j k}=(-1)^{j+k} \bar{W}\left(\begin{array}{l}
11 j \\
11 k
\end{array}\right)=\left|\begin{array}{ccc}
1 / 3 & 1 / 3 & 1 / 3 \\
1 / 3 & 1 / 6 & -1 / 6 \\
1 / 3 & -1 / 6 & 1 / 30
\end{array}\right| \text {, }
$$

we can finally write the geometrical factor $\left(6^{\prime}\right)$, as a function of the observable variables of polarization and direction, in the form

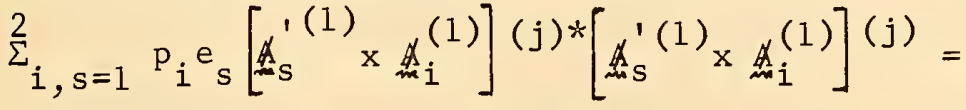

$$
\begin{aligned}
& =(2 j+1) \quad \Sigma_{k}{ }_{j k} R_{k} \text {. }
\end{aligned}
$$

This formula reduces to (6) when $\mathrm{F}_{\ell}, \mathrm{P}_{c}, Q_{\ell}$, and $Q_{c}$ vanish and $\xi=2$. 
Substitution into (4) of the geometrical factor thus evaluated yields the cross section for detection of the scattered radiation, by an analyzer of given characteristics, in the form

$$
\begin{aligned}
\frac{d \sigma}{d \Omega} & =c \sum_{j k}\left|\left(I_{f}\|p[j]\| I_{i}\right)\right|^{2} M_{j k} \xi_{k} R_{k}\left(P_{l}, P_{c}, Q_{l}, Q_{c}, \psi, \theta, \varphi\right) \\
& =\sum_{k=0}^{2} c_{k} R_{k}
\end{aligned}
$$

Several points may be emphasized regarding this formula:

1) The zero-degree coefficient $c_{0}$ is 3 times the average value of the cross section over all the orientation variables. This was expected since the $\Sigma_{k}$ represents an expansion into harmonic functions of the orientations.

2) When all polarization effects are absent or are averaged out, the experimental dependence of the cross section on $\cos ^{2} \theta$ determines the value of the coefficient $\mathrm{c}_{2}$.

3) Once $c_{2}$ is thus determined, the dependence of the cross section on all the linear polarization variables is completely determined through the expression (9) of $\mathrm{R}_{2}$. Therefore: a) the observation of linear polarization effects contributes no independent information on the scattering process, and b) conversely, the properties of polarization. considered in this section enable one to utilize the scattering process as a quantitative polarizer or analyzer tool once $c_{2}$ has been determined.

4) Experimental determination of $c_{1}$ requires the presence of circular polarization in the incident beam and the measurement of circular polarization of the scattered radiation. 
5) Experimental determination of all three coefficients $c_{0}, c_{1}$, and $c_{2}$ (or equivalent information) is necessary and sufficient to determine the magnitudes of the three $2^{j}$-pole polarizabilities, by inversion of (12), which yields

$$
\left|\left(I_{i}\left\|F^{[j]}\right\| I_{f}\right)\right|^{2}=\frac{1}{C \xi} \Sigma_{k}\left(M^{-1}\right)_{j k} c_{k}
$$

where

$$
\left(M^{-1}\right)_{j k}=(-1)^{j+k}(2 j+1)(2 k+1) \quad \bar{W}\left(\begin{array}{l}
11 j \\
11 k
\end{array}\right)=\left|\begin{array}{ccc}
1 / 3 & 1 & 5 / 3 \\
11 / 3 & -5 / 2 & -5 / 2 \\
5 / 3 & -5 / 2 & 5 / 6
\end{array}\right| \text {. }
$$

6) All three coefficients $c_{k}$ are equal when the vector and quadrupole polarizabilities vanish.

\section{POLARIZABILITY ANALYSIS IN TERMS OF INTERMEDIATE STATES}

We shall now consider the connection bolween the $2^{j}$-pole polarizabilities $\left(I_{f} \| P\left[j I^{[j}\right)\right.$, which determine the $v$-ray scattering, and other nuclear parameters. As is well known, dispersion relations tie the electric polarizability of a system to its absorption of radiation by electric dipole interaction. The usual dispersion relations pertain to scalar polarizability; but in our problem we deal also with a vector and a quadrupole polarizability. We should, therefore, derive a more general dispersion relation.

Since $\underline{m}^{[j]}=\left[\underline{x}^{[1]} \times\left(0_{-}+(-1)^{j} O_{+}\right) \underline{m}^{[1]}\right][j]$, its reduced matrix elements $\left(T_{f} \| P[j] I_{i}\right)$ can be expressed in terms of the reduced matrix elements of the dipole operator $\mathrm{r}^{[j]}$ pertaining to transitions between the states $I_{i}$ or $I_{f}$ and an intermediate state. The intermediate state can be classified by $i$ ts angular momentum quantum number $I=I_{i}+1, I_{i}$, or $I_{i}-1$, and by an additional quantum number $\mathrm{n}$ which may be a continuous variable. 
The energy of this state will be indicated by $\mathrm{E}_{\mathrm{nI}}$. The intermediate state is an eigenstate of the operators $\mathrm{O}_{-}$and $\mathrm{O}_{+}$which become now algebraic functions of $\mathrm{E}_{\mathrm{nI}}$. With these conventions, (15.15) of FR59 yields the following expression of the polarizabilities:

$$
\begin{aligned}
& \left(I_{f}\left\|P^{[j]}\right\| I_{i}\right)= \\
& =(-1)^{I_{i}+I_{f}^{+j}}(2 j+1)^{\frac{1}{2}} \Sigma_{n I}\left(I_{f}\left\|^{r}[1]\right\| n I\right)\left[\frac{1}{E_{n I}-E_{i}-\hbar \omega}+\frac{(-1)^{j}}{E_{n I}-E_{i}+\hbar \omega}\right] \\
& x\left(n I\left\|r{ }^{[1]}\right\| I_{i}\right) \quad \bar{W}\left(\begin{array}{rrr}
I_{i} I_{i} j \\
1 & 1 & I
\end{array}\right)
\end{aligned}
$$

This is a Kramers-Heisenberg dispersion formula, generalized through the $\bar{W}$ factor to represent vector and quadrupole polarizabilities besides the scalar one. (In the scalar case, $j$ vanishes and $\bar{W}$ takes a simple form.) This formula provides little qualitative information unless it is discussed from the standpoint of a model that provides some assumption regarding the matrix elements and the energy levels $\mathrm{E}_{\mathrm{n} I}$. The coefficients $\bar{W}$ can be taken from tables ${ }^{5}$.

Some simplification of (14) is attained for elastic scattering, when $I_{f}=I_{i}, \omega^{\prime}=\omega$. The reduced matrix element can then be expressed in terms of the oscillator strength of the transition $I_{i} \rightarrow n I$ (averaged over magnetic quantum numbers)

\footnotetext{
5 a.Simon, J. H. Vandersluis, and L. C. Biedenharn, Tables of the Racah coefficients, Oak Ridge Nat. Lab. Report 1679 Special (1954).

b) M. Rotenberg, R. Bivins, N. Metropolis, and J. K. Wooten, The $3 j$ and 6 j Coefficients. Technology press, Cambridge, Mass. (1959). See particularly eqs. (2.22-25) for our application.
} 


$$
\begin{aligned}
& \left.\left(I_{f}\left\|r r^{[1]}\right\| n I\right)\left(n I \| r[1]_{\| I_{i}}\right)=(-1)^{I_{i}+1-I} \mid(n I \| r]^{[1]} \| I_{i}\right)\left.\right|^{2}= \\
= & (-1)^{I_{i}+1-I} 3\left(2 I_{i}+1\right) \frac{\hbar^{2}}{2 M\left(E_{n I}-E_{i}\right)}\left\langle f_{n I, I_{i}}\right\rangle,
\end{aligned}
$$

where $M$ indicates the proton mass. When $I_{f}=I_{i}$, the $\bar{W}$ coefficient in (14) is the same as one finds in the expression of the $2 j$-pole interaction energy between systems with quantum numbers $I_{i}$ and 1 coupled with resultant quantum number I. Ref. 5a, P. XIII-XIV, also provides a simplified form of this $\bar{W}$ coefficient which can be cast in the form

$$
\bar{W}\left(\begin{array}{ccc}
I_{i} & I_{i} j \\
1 & 1 & I
\end{array}\right)=\frac{(-1)^{I_{i}+I+1}}{\left[3\left(2 I_{i}+1\right)\right]^{1 / 2}} \quad F_{j}\left(I_{i}, I\right),
$$

where

$$
F_{j}\left(I_{i}, I\right)= \begin{cases}\frac{1}{I(I+1)-I_{i}\left(I_{i}+1\right)-2}, & \text { for } j=0 \\ {\left[8 I_{i}\left(I_{i}+1\right)\right]^{1 / 2}} & \text { for } j=1 \\ \frac{6\left[I(I+1)-I_{i}\left(I_{i}+1\right)-2\right]\left[I(I+1)-I_{i}\left(I_{i}+1\right)-1\right]-16 I_{i}\left(I_{i}+1\right)}{160\left[I_{i}\left(I_{i}+1\right)\left(2 I_{i}-1\right)\left(2 I_{i}+3\right)\right]^{1 / 2}} & \text { for } j=2 .\end{cases}
$$

Substitution of (15) and (16) into (14) yields

$$
\begin{aligned}
& \left(I_{i} \| P[j]_{\left.\| I_{i}\right)=}\right. \\
& =(-1)^{j}\left[3\left(2 I_{i}+1\right)(2 j+1)\right]^{1 / 2} \Sigma_{I_{j}} F_{i}\left(I_{i}, I\right)\left\{\sum_{n} \frac{h^{2}}{2 M\left(E_{n I}-E_{i}\right)}\left\langle E_{n I, I_{i}}\right\rangle\right. \\
& \left.\times\left[\frac{1}{E_{n I}-E_{i}-\hbar \omega}+\frac{(-1)^{j}}{E_{n I}-E_{i}+\hbar \omega}\right]\right\}
\end{aligned}
$$


Whereas the usual Kramers-Heisenberg dispersion formula provides a single equation, which relates the scalar polarizability to a function of the distribution of oscillator strengths, we have now two additional equations, which relate the vector and quadrupole polarizabilities to two different functions of the oscillator strength distribution. Experimental determination of the three polarizabilities, as functions of $\boldsymbol{\omega}$, yields then additional information on the distribution of oscillator strength. More specifically, it is known that the KramersHeisenberg formula can be inverted, at least in principle, to determine the spectral distribution of oscillator strength, $\Sigma_{I}\left\langle f_{n I, I_{i}}\right\rangle$. Since the contributions of three values of $I$, namely $I_{i}$ and $I_{i} \pm 1$, are pooled in this spectral distribution, one may inquire whether the availability of data on the three polarizabilities, with $j=0,1,2$, would enable us to sort out the contributions of the transitions to states with different $I$. This would be possible if the expression in the braces of (18) were a function of $I$ but not of $j$, since (18) would then constitute a system of three linear equations in the three unknown values of the braces for $I=I_{i}, I_{i} \pm 1$. However, the braces include a factor $(-1)^{j}$ so that (18) includes in fact six separate functions of $I$, namely $\Sigma_{n}\left\langle f_{n I, I_{i}}\right\rangle \hbar^{2} / 2 m\left(E_{n I}-E_{i} \pm \hbar \omega\right)$ for three values of $I$ and two alternative signs in the last factor.

5. POLARIZABILITY ANALYSIS IN THE BOHR-MOTTELSON MODEL

It is a characteristic property of the states of a rotational band that the reduced matrix elements of a tensorial operator between different pairs of states of the band are proportional to a single matrix 
element, evaluated in the "intrinsic" coordinate system. ${ }^{6}$ The proportionality coefficients are determined by the following geometric considerations.

In the intrinsic coordinate system consider the ground state of the nucleus, call $\mathrm{K}_{i}$ its (non-negative) angular momentum quantum number about the $z$ axis of this system, and indicate this state by $\left(K_{i}\right)$. To this state corresponds in the laboratory system a band of rotational states indicated by $\left(\mathrm{K}_{i} \mathrm{I}_{i} \mathrm{~m}_{i}\right)$, which are represented in terms of $\left.\mid \mathrm{K}_{i}\right)$ and of wave functions of the symmetrical top $D^{\left(I_{i}\right)} K_{i} m_{i}(\psi, \theta, \varphi)$. Similarly a tensorial set of operators $t[k]$, such as the set of components of the $2^{k}$-pole polarizability, is represented in the laboratory system by $\Sigma_{q^{\prime}} t^{[k]} q^{\prime} D_{q^{\prime} q}^{(k)}(\psi, \theta, \varphi)$, where $t^{[k]}$ indicates an operator of the set in the intrinsic system. It follows that a matrix element

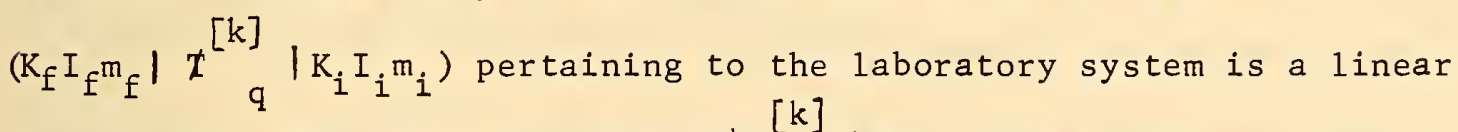
combination of matrix elements $\left(K_{f}\left|t^{[k]} q^{\prime}\right| K_{i}\right)$ of the intrinsic system, whose coefficients are integrals over $D$ functions expressed in terms of Wigner coefficients. (Notice, however, that this procedure is meaningful only insofar as the operator $\mathrm{T}_{\mathrm{T}}^{[\mathrm{k}]}$ is independent of the energy of the final state $\mathrm{K}_{f} \mathrm{I}_{\mathrm{f}} \mathrm{m}_{\mathrm{f}}$. In our problem this condition holds only in the approximation where $\omega^{\prime}$ in $O_{+}$may be taken equal to $\omega$, i.e. when $\hbar\left(\omega-\omega^{\prime}\right)=E_{f}-E_{i} \sim 0$. This assumption will be made in the following.)

\footnotetext{
$\overline{6}$ A. Bohr and B. R. Mottelson, Dan. Mat. Fys. Medd., 27, No. 16 (1953), p. 109 .
} 
We shall be interested here in matrix elements between states of the same rotational band, in which case $\mathrm{K}_{f}=\mathrm{K}_{i}$ and $\left(\mathrm{K}_{\mathrm{f}}\left|\mathrm{t} \mathrm{q}^{\prime}\right| \mathrm{K}_{i}\right)$ vanishes unless $q^{\prime}=0$ or $q^{\prime}=2 K_{i}$. The matrix elements with $q^{\prime}=2 K_{i}$ arise insofar as the tensorial operator can reverse the sign of the spin in the intrinsic system. Considering that $\left|q^{\prime}\right| \leqslant k$, that $k \leqslant 2$ in our problem, that $\mathrm{K}_{i}=1$ does not occur in the lowest band of deformed nuclei, and that $\mathrm{K}_{i}=1 / 2$ occurs only in a few instances, we sha 11 disregard henceforth the contributions from $\mathrm{q}^{\prime}=2 \mathrm{~K}_{\mathrm{i}} \neq 0$.

Under this restrictive condition, all matrix elements $\left(K_{i} I_{f} m_{f}\left|t^{[k]}\right| K_{i} I_{i} m_{i}\right)$ are proportional to the single matrix element $\left(\mathrm{K}_{i}\left|\mathrm{~T}^{[\mathrm{k}]}\right| \mathrm{K}_{\mathrm{i}}\right)$ of the intrinsic system. Here we need only consider the reduced matrix elements $\left(\mathrm{K}_{i} I_{f}\left\|\mathrm{~T}^{[\mathrm{k}]}\right\| \mathrm{K}_{i} I_{i}\right)$, since the dependence of the matrix elements on the magnetic quantum numbers $\left(m_{i}, m_{f}\right.$ ) is given by the Wigner-Eckart theorem (FR59, Eq. (14.4)) which has been taken into account implicitly in Sect. 2. These matrix elements are given by

$$
\left(\mathrm{K}_{i} I_{f}\left\|T^{[k]}\right\| K_{i} I_{i}\right)=\sqrt{2 I_{i}+1}\left(I_{i} K_{i} k 0 \mid I_{i} \mathrm{kI}_{f} K_{i}\right)\left(K_{i}\left|T^{[k]}{ }_{0}\right| K_{i}\right) \text {. }
$$

In the case of the polarizability matrix of (3) and (4), this equation reads

$$
\begin{aligned}
& \left(K_{i} I_{f}\left\|P^{[j]}\right\| K_{i} I_{i}\right)= \\
= & \sqrt{2 I_{i}+1}\left(I_{i} K_{i} j 0 \mid I_{i} j I_{E} K_{i}\right)\left(K_{i}\left|\not_{0}^{[j]}\right| K_{i}\right) .
\end{aligned}
$$

A sum rule follows from this result, namely that the total cross section for elastic plus Raman scattering within a rotational band depends only on the polarizability in the intrinsic coordinate system: 


$$
\begin{aligned}
& \Sigma_{I_{f}}\left|\left(K_{i} I_{f} \| P[j]_{\| K_{i} I_{i}}\right)\right|^{2}= \\
= & \left(2 I_{i}+1\right)\left|\left(K_{i}\left|\not p[j]_{0}\right| K_{i}\right)\right|^{2} .
\end{aligned}
$$

Analysis in the intrinsic system. The analysis of polarizability in terms of dipole transitions to intermediate states, carried out in general form in Sect. 4, can also be carried out for the Bohr-Mottelson model in the intrinsic system. Here there is no further reason to utilize standard notation and we can express the polarizability operators in ordinary coordinates. However we keep the complex combinations of coordinates $x \pm i y$ because they lead to transitions from the ground state $\mathrm{K}_{\mathrm{i}}$ only to intermediate states $(\mathrm{n}, \mathrm{K})$ with $\mathrm{K}=\left|\mathrm{K}_{\mathrm{i}} \pm 1\right|$, respectively.

Translation. from standard notation to ordinary coordinates yields

$$
\begin{aligned}
& p^{[j]}=\left[\underset{m}{\underline{x}}[1] \times\left(0_{-}+(-1)^{j_{0}}\right){\underset{m}{x}}^{[1]}\right][j]{ }_{0}^{[j]}= \\
& \frac{1}{2 \sqrt{3}}\left[(x-i y)\left(0_{-}+0_{+}\right)(x+i y)+(x+i y)\left(0_{-}+0_{+}\right)(x-i y)+2 z\left(0_{-}+0_{+}\right) z\right] \text {, for } j=0 \text {, } \\
& \frac{1}{2 \sqrt{2}}\left[-(x-i y)\left(0_{-}-0_{+}\right)(x+i y)+(x+i y)\left(0_{-}-0_{+}\right)(x-i y)\right], \quad \text { for } j=1 \text {, } \\
& \frac{1}{2 \sqrt{6}}\left[(x-i y)\left(0_{-}+0_{+}\right)(x+i y)+(x+i y)\left(0_{-}+0_{+}\right)(x-i y)-4 z\left(0_{-}+0_{+}\right) z\right] \text {, for } j=2 \text {. }
\end{aligned}
$$

Indicating the intermediate states by $\mathrm{nK}$ we have, then, 


$$
\begin{aligned}
& \left(K_{i}\left|p_{0}^{[0]}\right| K_{i}\right)=\frac{1}{\sqrt{3}} \Sigma_{n}\left[\frac{E_{n, K_{i}+1-E_{0}}}{\left(E_{n, K_{i}+1}-E_{0}\right)^{2}-\hbar^{2} \omega^{2}}\left|\left(n, K_{i}+1|x+i y| K_{i}\right)\right|^{2}+\right. \\
& +\frac{E_{n,\left|K_{i}-1\right|}-E_{0}}{\left(E_{n,\left|K_{i}-1\right|}-E_{0}\right)^{2}-\hbar^{2} \omega^{2}}\left|\left(n, K_{i}-1|x-i y| K_{i}\right)\right|^{2}+ \\
& \left.+\frac{E_{n K_{o}}-E_{K_{o}}}{\left(E_{n K_{0}}-E_{0}\right)^{2}-\hbar^{2} \omega^{2}} 2\left|\left(n K_{i}|z| K_{i}\right)\right|^{2}\right]
\end{aligned}
$$

$$
\begin{aligned}
& \left(K_{i}\left|p_{0}^{[1]}\right| K_{i}\right)=-\frac{1}{\sqrt{2}} \sum_{n}\left[\frac{\hbar^{2} \omega^{2}}{\left(E_{n, K_{i}+1}-E_{0}\right)^{2}-\hbar^{2} \omega^{2}}\left|\left(n, K_{i}+1|x+i y| K_{i}\right)\right|^{2}-\right. \\
& \left.-\frac{\hbar^{2} \omega^{2}}{\left(E_{n,\left|k_{i}-1\right|}-E_{0}\right)^{2}-\hbar^{2} \omega^{2}}\left|\left(n,\left|k_{i}-1\right||x-i y| k_{i}\right)\right|^{2}\right],
\end{aligned}
$$

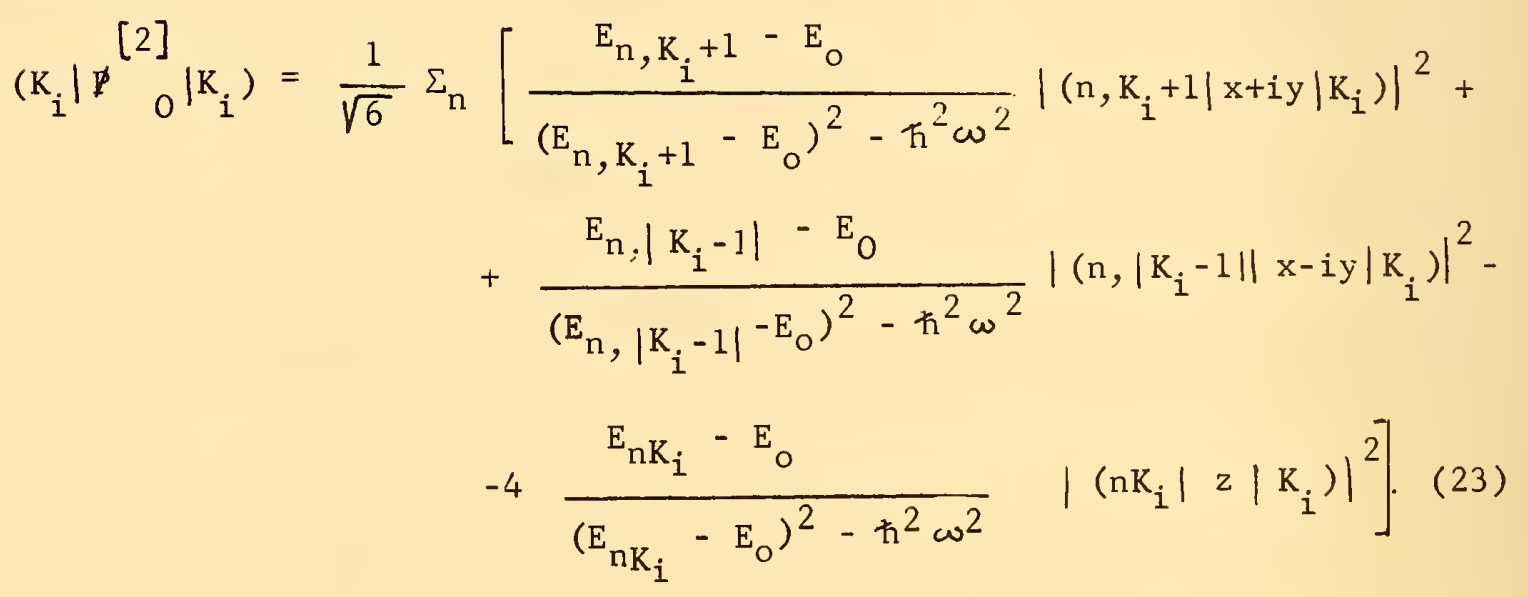


Notice that the matrix element of $\not 1]$ (vector polarizability) vanishes unless the excitation spectra to states with $K=\left|K_{i} \pm 1\right|$ differ. No indication of such a difference has yet been detected for giant resonance transitions, and none could occur for the even-even nuclei in which $k_{i}=0$ and $\left|k_{i}-1\right|=k_{i}+1$. Similarly the matrix element of $\not 2]$ (quadrupole polarizability) vanishes unless there is a difference between transverse and longitudinal excitations. A probable cause of similarity between the spectra with $\mathrm{K}=\left|\mathrm{K}_{i} \pm 1\right|$ is indicated in the next Section.

\section{CORE POLARIZABILITY IN THE PRESENCE OF A "SPECTATOR" PARTICLE}

Situations occur in which a nucleus may be regarded as consisting of a single particle and of a more symmetrical core which are weakly coupled. In this event the nuclear polarizability should be primarily determined by the large core and should exhibit the core's symmetry. Examples of this kind may be the $\mathrm{Bi}_{83}^{209}$ nucleus with a single proton outside the spherical "double-magic" $\mathrm{Pb}_{82}^{208}$ core, and any odd-A deformed nucleus in which the elongated spinless residue of mass A-1 is presumably unaffected by any reversal of the direction of rotation of the odd particle. It will be shown here how, when such conditions prevail, the polarizability of the whole nucleus reduces to the polarizability of the core and exhibits the core's symmetry.

In a $\mathrm{Bi}_{83}^{209}$-type nucleus, the ground state may be represented by the coupling scheme symbol $\left(0, I_{i} ; I_{i}\right)$, where 0 is the angular momentum of the core, and $I_{i}$ indicates both the angular momentum of the odd proton and the total angular momentum of the whole nucleus. Similarly a 
state obtained by electric dipole excitation of the core, with spin $I=I_{i}-1, I_{i}$, or $I_{i}+1$, may be represented by $\left(n 1, I_{i} ; I\right)$. The reduced matrix element of the dipole operator $\underset{\mathrm{m}}{\mathrm{r}}$ between these two nuclear states can be expressed in terms of the corresponding matrix element for the core alone by the projection formula (15.7) of FR59.

$$
\begin{aligned}
& \left(n 1, I_{i} ; I\left\|r^{[1]}\right\| 0, I_{i} ; I_{i}\right)=(-1)^{2 I_{i}}\left(n 1\left\|r^{[1]}\right\| 0\right)\left[\left(2 I_{i}+1\right)(2 I+1)\right]^{1 / 2} \\
& \times \quad \bar{W}\left(\begin{array}{lll}
I & I_{i} & 1 \\
0 & 1 & I_{i}
\end{array}\right)= \\
& =(-1)^{I-I_{i}+1}(2 I+1)^{1 / 2} \sqrt{\frac{1}{3}}\left(n 1\left\|r^{[1]}\right\| 0\right)
\end{aligned}
$$

where the dependence on $I$ is reduced to the weight and phase factor

$$
(-1)^{I-I_{i}+1}(2 I+1)^{1 / 2} \text { If this result is entered in the polarizability }
$$

formula (14), with $I_{f}=I_{i}$, and if the weak coupling approximation is made, $E_{n I}=E_{n 1}$ independently of $I$, the sum over $I$ can be carried out analytically and the polarizability is seen to vanish for $j \neq 0$. That is, the spherically symmetric core contributes no vector or tensor polarizability, as was to be expected. (To verify this result, one must take into account the conjugation property of the reduced matrix element (24), namely Eq. (14.8) of FR59, and a special case of the orthonormality Eq. (11.15) of FR59, namely $\left[3\left(2 I_{i}+1\right)\right]^{1 / 2} \Sigma_{i}(2 I+1)(-1)^{I_{i}+1+I} \bar{W}\left(I_{i} I_{i} j / 11 I\right)=$ $\left.\oint_{\text {jo }}.\right)$

In an odd-A deformed nucleus, the ground state with spin $\mathrm{K}_{i}$ may be represented, in the intrinsic coordinate system, by the coupling scheme 
symbol $\left(0, \mathrm{~K}_{\mathbf{i}} ; \mathrm{K}_{\mathbf{i}}\right)$, in which 0 indicates the spin of the elongated residue of mass $A-1$ and the first index $K_{i}$ represents the angular momentum of the odd particle. A state obtained by transverse electric dipole excitation of the spinless residue may be represented by $\left(n 1, K_{i} ; K_{i} \pm 1\right)$. Insofar as the odd particle is weakly coupled to the residue, the excitation energy and the dipole matrix element of the transition will be independent of whether the transition induces an angular momentum parallel or opposite to that of the odd particle, i.e. whether their resultant is $k_{i}+1$ or $k_{i}-1$. In this event the vector $(j=1)$ polarizability will vanish, according to (23), as it would do for an even-A spinless nucleus.

\section{APPENDIX}

\section{VECTOR DIAGRAM OF THE BIEDENHARN RECOUPLING IDENTITY}

The connection between the ordinary angular correlation formula and the formulas obtained by emphasizing the angular momentum transferred in the scattering process is illustrated by Figure 2. In this figure is reproduced the same pair of vector triangles, $\left(I_{i}, L_{m}, I\right)$ and $\left(I_{m}, L_{m}, I_{m f}\right)$, as was shown in Figure 1b. Consider now that the directions of $I_{m}, L_{m i}$, and $L_{m}$, are not identified in the scattering process; indeed

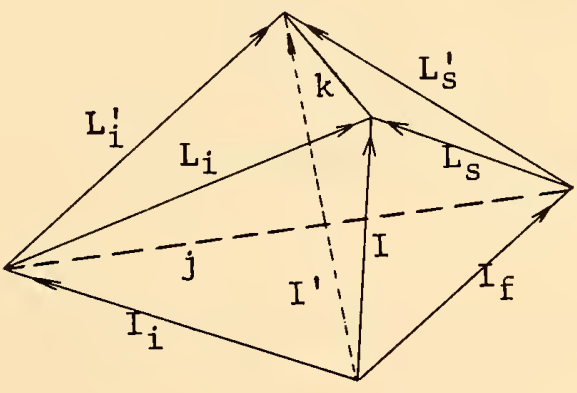

Figure 2 even the magnitude of I is not identified, and those of $\mathrm{L}_{m i}$ and $\mathrm{L}_{m}$ 
would vary if the limitation to dipole transitions were relaxed. The interference effects arising from alternative vectors $\underset{m}{I}, L_{m i}$, and ${\underset{m}{S}}_{S}$ are represented by adding to the diagram a second pair of triangles, $\left(I_{i}, L_{m i}^{\prime}, I_{m}^{\prime}\right)$ and $\left(I_{m}^{\prime}, L_{S^{\prime}}^{\prime}, I_{m f}\right)$. The possible magnitudes of the vector $k=L_{i}-L_{i}^{\prime}=L_{s}^{\prime}-L_{s}^{\prime}$, namely 0,1 , or 2 , correspond to the degrees of the various terms in the harmonic analysis of the distribution of scattered radiation (Chap. 19 of FR59). The 9 vectors 1 isted thus far form a triangular bipyramid consisting of two tetrahedra with the common base $\left(\mathrm{I}, \mathrm{I}^{\prime}, \mathrm{k}\right)$. The edges of these two tetrahedra correspond, respectively, to the indices of the two $\bar{W}$ coefficients that appear in a scattering formula constructed on the pattern of Eq. (19.11) of FR59.

The angular momentum transfer $j$ is represented by the diagonal that joins the opposite vertices of the bipyramid. The vector triangles $\left(I_{i}, j, I_{m f}\right)$ and $\left(L_{i}, j, L_{m s}\right)$ are the same as are shown in Figure la. Notice that these two triangles, together with $\left(\mathrm{L}_{i}^{\prime}, \mathrm{m}_{\mathrm{m}}^{\mathrm{j}} \mathrm{L}_{\mathrm{s}}^{\prime}\right)$, subdivide the bipyramid into three tetrahedra with the common edge $j$. The edges of the tetrahedron $\left(L_{i}, L_{i}^{\prime}, L_{s}, L_{s}^{\prime}, j, k\right)$ correspond to the indices of the $\bar{W}$ coefficient in ( 7$)$, those of $\left(\mathrm{I}_{\mathrm{i}}, \mathrm{L}_{\mathrm{i}}, \mathrm{I}_{\mathrm{f}}, \mathrm{L}_{\mathrm{s}}, \mathrm{I}_{\mathrm{m}}, \mathrm{j}_{\mathrm{m}}\right)$ to the indices of the $\bar{W}$ in (14), those of the third tetrahedron to the indices of the additiona $1 \bar{W}$ that appears when the square of (14) is substituted into (4).

Altogether, the edges of the five tetrahedra that can be seen in Figure 2 correspond to the indices of the five $\overline{\mathrm{W}}$ coefficients of the Biedernharn identity in (19.12) of FR59. The identity of a product of 
two $\bar{W}$ and of a sum of products of three $\bar{W}$ relates to the fact that the bipyramid can be subdivided alternatively into two or into three tetrahedra . 

U.S. DEPARTMENT OF COMMERCE

Frederick H. Mueller, Secretary

NATIONAL BUREAU OF STANDARDS

A. V. Astin, Director

\section{THE NATIONAL BUREAU OF STANDARDS}

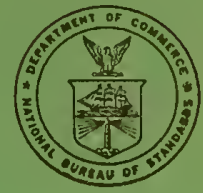

The scope of activities of the National Bureau of Standards at its major laboratories in Washington, D.C., and Boulder:, Colo., is suggested in the following listing of the divisions and sections engaged in technical work. In gencral, each section carries out specialized research, development, and engineering in the field indicated by its title. A brief description of the activities, and of the resultant publications, appears on the inside of the front cover.

WASHINGTON, D.C.

ELECTRICITY. Resistance and Reactance. Electrochemistry. Electrical Instruments. Magnetic Measurements. Diclectrics.

METROLOGY. Photometry and Colorimetry. Refractometry. Photographic Research. Length. Engineering Metrology. Mass and Scale. Volumetry and Densimetry.

HEAT. Temperature Physics. Heat Measurements. Cryogenic Physics. Rheology. Molecular Kinetics. Free Radicals Research. Equation of State. Statistical Physics. Molecular Spectroscopy.

RADIATION PHYSICS. X-Ray. Radioactivity. Radiation Theory. High Energy Radiation. Radiological Equipment. Nucleonic Instrumentation. Neutron Physics.

CHEMISTRY. Surface Chemistry. Organic Chemistry. Analytical Chemistry. Inorganic Chemistry. Electrodeposition. Molecular Stıucture and Properties of Gases. Physical Chemistry. Thermochemistry. Spectrochemistry. Pure Substances.

MECHANICS. Sound. Pressule and Vacuum. Fluid Mechanics. Engineering Mechanics. Combustion Controls. ORGANIC ANí FIBROUS MATERIAIs. Rubber. Textiles. Paper. Leather. Testing and Specifications. Polymer Structure. Plastics. I)ntal Research.

METALLURGY. Ther'mal Metallurgy. Chemical Metallurgy. Mechanical Metallurgy. Corrosion. Metal Physics. MINERAL PRODUCTS. Engineering Ccramics. Glass. Refractories. Enameled Metals. Constitution and Microstructure.

BUILDING RESEARCH. Structural Engincering. Fire Research. Mechanical Systems. Organic Building Materials. Codes and Safety Stanclards. Heat Transfer. Inorganic Building Materials.

APPLIED MATHEMATICS. Numerical Analysis. Computation. Statistical Engineering. Mathematical Physics.

DATA PROCESSING SYSTEMS. Components and Techniques. Digital Circuitry. Digital Systems. Analog Systems. Applications Engineering.

ATOMIC PHYSICS. Spectioscopy. Radiometry. Mass Spectrometry. Solid State Physics. Electron Physics. Atomic Physics.

INSTRUMENTATION. Engineering Electronics. Electron Devices. Electronic Instrumentation. Mechanical Instruments. Basic Instrumentation.

Office of Wcights and Measures.

BOULUER, COLO.

CliYOGENIC ENGINEERING. Cryogenic Fquipment. Cryogenic Processes. Properties of Materials. Gas Liţuefaction.

IONOSPHERE RESEARCH AND PIROPAGATION. Low Frequency and Very Low Frequency Research. Ionosphere Iescarch. Prediction Services. Sun-Earth Relationships. Field Engineering. Radio Warning Sel:vices. RAIIO PROPAGATION ENGINEEIRING. Data Reduction Instrumentation. Radio Noise. Tropospheric Measurcments. Tropospheric Analysis. Propagation-Terrain Effects. Radio-Meteorology. Lower Atmosphere Physics. RADIO STANDARIDS. High frequency Electrical Standards. Radio Broadcast Service. Radio and Microwave Materials. Atomic Frequency and Time Standards. Eilectronic Calibration Center. Millimeter-Wave Research. Miciowave Circuit Standlards.

RAI)IO SYSTEMS. High Frequency and Very High Frequency Research. Modulation Resear'ch. Antenna Rescal:ch. Navigation Systems. Space Telecommunications.

UPPER ATMUSPHERE AND SPACE PHYSICS. Upper Atmosphere and Plasma Physics. Ionosphere and Exosphere Scatter. Airglow and Aurora. Ionospheric Radio Astronomy. 
NBS 